\title{
Dynamique de croissance en relation avec le potentiel hydrique foliaire chez deux espèces fourragères
}

Basile NOÏTSAKIS

Laboratoire de Science des Pâturages (236), Université de Thessalonique, 54006 Thessalonique, Grèce

RÉSUMÉ

\begin{abstract}
Deux espèces de Graminées fourragères, Dichanihium ischaemum (L.) Roberty et Chrysopogon gryllus Trin, ont été cultivées sous 2 régimes hydriques contrastés, afin d'étudier $a$ ) les propriétés hydrodynamiques des feuilles ; $b$ ) l'influence du potentiel hydrique foliaire sur la dynamique de croissance des 2 espèces.

Les observations ont montré, que l'indice foliaire et le taux d'élongation du limbe sont assez sensibles au potentiel hydrique, avec des répercussions analogues sur le taux de croissance de la biomasse aérienne. Toutefois, le Dichanthium s'avère moins sensible au déficit de saturation hydrique, et sa productivité au niveau des feuilles semble être moins affectée par rapport à celle de Chrysopogon.

Ce comportement comparatif des 2 espèces dans leur réponse à la contrainte hydrique nous permet d'envisager une organisation rationnelle de l'exploitation pastorale des régions sèches.
\end{abstract}

Mots clés additionnels : Contrainte hydrique, production fourragère, Dichanthium ischaemum, Chrysopogon gryllus.

Growth dynamics in relation to xylem water potential in two forage species.

Two forage grasses Dichanthium ischaemum (L.) Roberty and Chrysopogon gryllus Trin were cultivated under water stress conditions in order to study internal water relations as well as the response of leaf area index and leaf elongation ratio 10 water deficit. Both parameters were affected by water deficil, producing a proportional decrease in relative growth rate. However differences in leaf elongation, leaf area index and relative growth rate were observed between the two species. As leaf water potential decreased, the relative growth rate of leaves was inhibited earlier in Chrysopogon than in Dichanthium. This different response of the two species to water deficit is a sensitive basis for scoring the incidence of water stress and provides a useful tool in screening for drought resistance.

Additional key words : Water deficit, forage production, Dichanthium ischaemum, Chrysopogon gryllus.

\section{INTRODUCTION}

Des périodes de sécheresse estivale, en relation avec une pluviométrie assez faible et/ou des niveaux élevés de températures sont fréquentes dans les régions méditerranéennes. L'histoire du déficit hydrique développé dans les tissus des plantes est rarement prise en compte, quand les réponses métaboliques sont analysées (KING \& BUSH, 1985). En outre, on ne sait pas clairement de quelle façon la contrainte hydrique réagit sur la production de biomasse aérienne (HSIAO et al., 1976 ; TURNER, 1979).
On peut considérer que l'élongation du limbe et l'importance de la surface foliaire constituent les principales composantes de la production primaire chez les plantes fourragères (HOVELAND et al., 1974; HORST et al., 1978). L'augmentation des surfaces foliaires dépend de processus physiologiques soumis à l'influence de la contrainte hydrique (HSIAO, 1973 ; BOYer, 1970 ; BOYER \& MC PHERSON, 1975 ; BEGG \& TURNER, 1976 ; TURNER, 1979). D'ailleurs, la croissance volumique des cellules foliaires est un processus physiologique très sensible à la contrainte hydrique (HSIAO, 1973). De même l'extension de la surface foliaire est affectée par la contrainte hydrique (THO- 
MAS et al., 1976). LUDLOW \& NG (1977) ont également observé que l'élongation du limbe chez Panicum maximum Jacq, cesse lorsque le potentiel hydrique descend à - 1,1 MPa. CutLer et al. (1980) ont trouvé que cette élongation chez Oryza sativa L. cesse à $-0,6 \mathrm{MPa}$ pour les plantes irriguées et entre - 1,0 MPa et - 1,2 $\mathrm{MPa}$ pour les plantes sous contrainte hydrique.

Pour les plantes fourragères, un assez petit nombre d'études sont disponibles, concernant l'influence de la contrainte hydrique sur les paramètres de la croissance (WOLF \& PARRISH, 1982 pour la Festuca arundinacea L.).

Cette contrainte hydrique est exprimée généralement au moyen de 2 paramètres, traduisant l'état hydrique de la feuille; le $1^{\mathrm{er}}$, le potentiel hydrique foliaire, traduit l'état énergétique de l'eau dans la feuille ; le $2^{\mathrm{e}}$, le déficit de saturation hydrique, se réfère plutôt à la quantité d'eau présente dans la feuille (TURNER, 1981). Dans l'étude présentée ici, on a essayé d'établir des relations entre le potentiel hydrique des feuilles et la croissance des parties aériennes chez 2 espèces : Dichanthium ischaemum (L.) Roberty et Chrysopogon gryllus (Trin), connues comme plantes fourragères de saison chaude. On a mis l'accent sur la sensibilité différente des 2 espèces au déficit hydrique, tout en faisant ressortir des conclusions valables pour une exploitation plus efficace des parcours pâturés.

\section{MATÉRIEL ET MÉTHODES}

\section{A. Matériel}

Les 2 espèces de graminées $D$. ischaemum et $C$. gryllus, sont cultivées dans des pots de $30 \times 40 \mathrm{~cm}$, sur le terrain expérimental du laboratoire de Science des pâturages de l'Université de Thessalonique, dans les conditions naturelles. Les plantes sont issues de grandes touffes éclatées, récoltées dans la région de Thessalonique. Pour mesurer les paramètres de production, au cours de la période végétative, on a combiné 2 facteurs (plante et régime hydrique) à 2 niveaux, en 4 répétitions avec 5 pots par mesure. Par conséquent, on a finalement étudié 80 pots aléatoirement répartis dans le dispositif expérimental. On s'est efforcé d'utiliser des plantes présentant, en début d'expérience, une bonne homogénéité de surface foliaire.

\section{B. Régimes hydriques}

Un an après la plantation et au début de la période végétative, 2 régimes hydriques étaient appliqués. Dans le premier, les plantes étaient en conditions d'irrigation optimale, c'est-à-dire que l'humidité du sol était au niveau de la capacité au champ (plantes irriguées). On rajoutait manuellement une quantité d'eau de l'ordre de 2,0 kg par pot, dès que la valeur moyenne des 4 hygromètres (MEGO-AFẺK soil moisture indicator) enfoncés dans le sol à la profondeur de $35 \mathrm{~cm}$, dépassait l'indication 25, qui correspond à un potentiel hydrique du sol de $0,15 \mathrm{MPa}$ environ.
Pour le $2^{\mathrm{e}}$ régime hydrique, les pots étaient en condition de contrainte hydrique (plantes stressées), c'està-dire que la quantité d'eau rajoutée était égale à $1 / 3$ de celle apportée sur les plantes irriguées. L'arrosage était réalisé à la même date pour les 2 régimes hydriques, à des intervalles moyens de 5 jours en avril et de 3 jours en mai, juin et juillet.

Il faut signaler que la région où se trouve la station expérimentale se caractérise par un climat estival sec avec $450 \mathrm{~mm}$ de pluie par an, réparties surtout pendant l'hiver, l'automne et le printemps.

\section{Méthodes}

Pour mesurer la production de biomasse aérienne, les plantes étaient coupées à la surface du sol 5 fois au cours de leur cycle cultural. Chaque fois, on récoltait la production de 4 pots différents.

Le taux de croissance de la biomasse (T.C.B.), ou relative growth rate, était calculé selon les formules de RADFORD (1967).

$$
\text { T.C.B. }=\frac{\mathrm{d}(\log ) \mathrm{W}}{\mathrm{dt}}=\frac{1 \mathrm{dW}}{\mathrm{W} \frac{\mathrm{dt}}{}}
$$

(où $\mathrm{W}=$ poids des feuilles, $\mathrm{t}=$ nombre de jours)

$L$ 'indice foliaire (I.F.) était également calculé à partir de 5 pots comme le rapport entre la surface foliaire mesurée à l'aide d'un planimètre, et la surface du sol du pot. Le taux d'élongation du limbe (T.E.L.) était mesuré au cours d'une période 20 jours, au cours du mois de juin, sur des talles tirées au hasard, en excluant celles qui étaient en bordure de la touffe.

Tous les 3 ou 4 jours, on mesurait pour chaque espèce et chaque régime hydrique, la longueur de 5 feuilles qui étaient, au point de vue de l'ordre d'apparition, les deuxièmes feuilles de la talle. Les feuilles mesurées étaient disposées le long d'une fine tige en plastique, enfoncée dans le pot tout à côté de la talle. Trois marquages étaient effectués : 1 sur la feuille pour qu'elle soit bien repérée, un autre sur la tige à hauteur de la base du limbe, et un dernier sur la tige à hauteur de l'extrémité du limbe. Le T.E.L. était calculé, à partir de 5 feuilles, comme le rapport entre la distance des marquages successifs et le nombre de jours correspondants. En même temps, on mesurait le potentiel hydrique.

Pour mesurer le potentiel hydrique foliaire $\left(\Psi_{F}\right)$, on a suivi la méthode de la chambre à pression, développée par SCHOLANDER et al. (1965b), RITCHIE \& HINCKLEY (1975). En ce qui concerne le déficit de saturation hydrique dans les feuilles (D.S.H.) qui exprime le rapport entre le poids d'eau présent dans la feuille à un instant donné, au poids d'eau maximal qu'elle peut contenir, on suivait la méthode de CÁTSKY (1965), décrite par BARRS (1968). Les 2 paramètres étaient mesurés vers $14 \mathrm{~h} 30,7$ fois au cours de l'essai, et ils étaient exprimés sous forme de moyennes de 4 échantillons de feuilles prélevées. L'erreur standard était également calculée. On réglait les dates des mesures du potentiel hydrique $\Psi$ et du déficit de saturation hydrique D.S.H. de manière à les faire coïncider avec les dates de mesure du taux de croissance de la biomasse T.C.B. et de l'indice foliaire I.F. 


\section{RÉSULTATS ET DISCUSSION}

\section{A. Relations entre le déficit de saturation hydrique D.S.H. et le potentiel hydrique $\Psi_{F}$ chez les 2 espèces étudiées}

La variation saisonnière du déficit de saturation hydrique (D.S.H.) dans la feuille de Dichanthium et de Chrysopogon, est représentée dans la figure 1a, b. En condition d'irrigation optimale (fig. la) le D.S.H. augmente à partir de la fin avril chez les 2 espèces de manière presque identique, jusqu'à la fin juin, date à laquelle on peut alors observer une différence significative assez importante entre Dichanthium (16 p. 100) et Chrysopogon (21 p. 100). Sous contrainte hydrique (fig. 1b) l'augmentation saisonnière du D.S.H. s'avère plus rapide chez le Chrysopogon et atteint sa valeur extrême (17 p. 100) à la mi-juillet, tandis que chez Dichanthium elle est plus lente et ne dépasse pas 12 p. 100. Cette différence entre les 2 espèces quant au déficit hydrique foliaire peut être interprétée en examinant la relation qui existe entre le D.S.H. et le potentiel hydrique foliaire (fig. $2 a, b$ ), relation qui

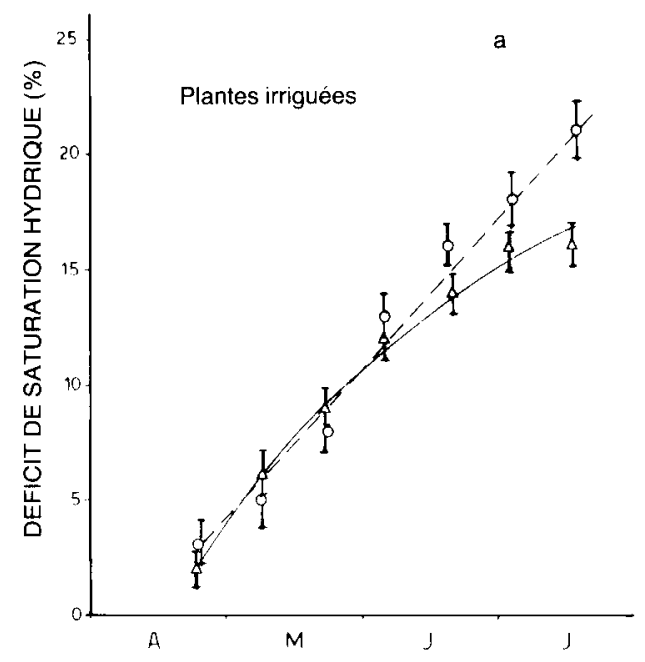

Figure 1

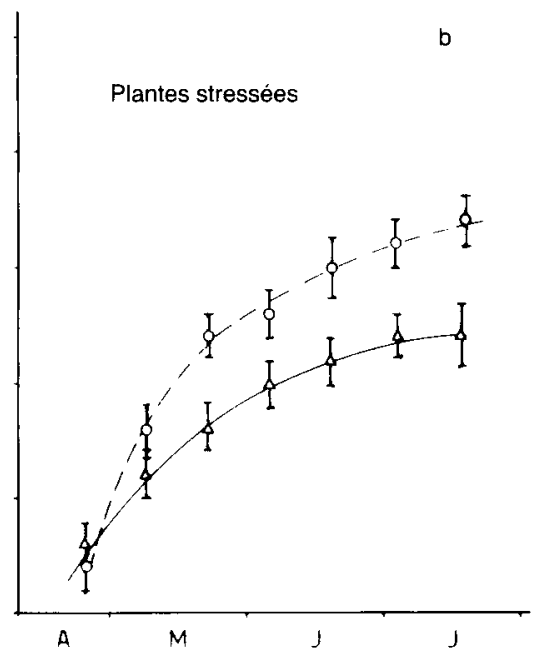

Evolution saisonnière $d u$ déficit de saturation hydrique chez Dichanthium ischaemum $(\Delta-\Delta)$ et chez Chrysopogon gryllus $(O-O)$. a. En condition d'irrigation optimale.

$b$. Sous contrainte hydrique.

Les traits verticaux indiquent l'erreur standard.

MOIS

Seasonal changes of water saturation deficit in Dichanthium ischaemum $(\Delta-\Delta)$ and Chrysopogon gryllus $(O-O)$.

a. Under optimum irrigation conditions.

b. Under water stress.

Figure 2

Evolution du déficit de saturation hydrique en fonction du potentiel hydrique foliaire chez Dichanthium ischaemum $(\Delta-\Delta)$ et chez Chrysopogon gryllus $(\mathrm{O}-\mathrm{O})$.

a. En condition d'irrigation optimale.

b. Sous contrainte hydrique.

Les mesures étaient effectuées les : 24/4, 8/5, 25/5, 5/6, 19/6, 3/7, $17 / 7$.

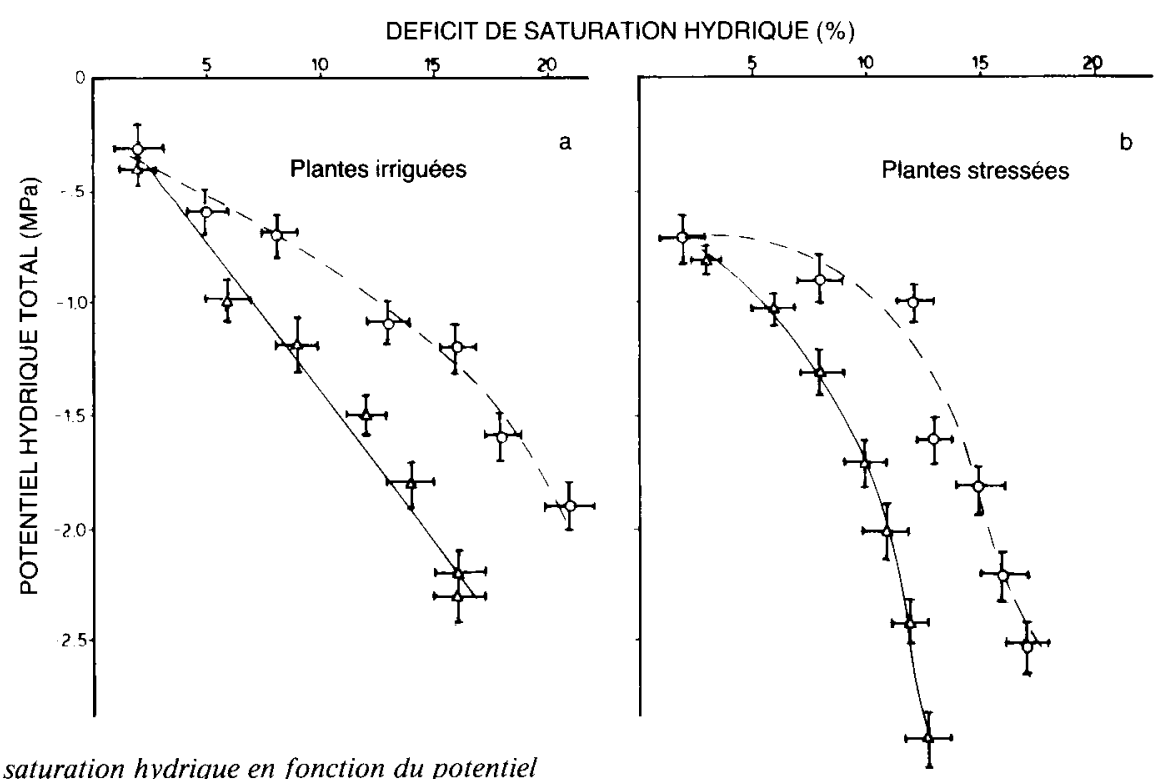

Water saturation deficit changes in relation to leaf water potential in Dichanthium ischaemum $(\Delta-\Delta)$ and Chrysopogon gryllus $(O-O)$. a. Under optimum irrigation conditions.

b. Under water stress.

Measurement dates : 24/4, 8/5, 25/5, 5/6, 19/6, 3/7, 17/7. 
traduit les propriétés hydrodynamiques de la feuille (BERGER, 1978). En effet il est admis que pour une valeur donnée du potentiel hydrique foliaire, le déficit de saturation hydrique chez les plantes résistantes à la sécheresse est plus faible que chez les plantes moins résistantes (SANCHEZ-DIAZ \& KRAMER, 1971 ; LUDLOW, 1976 ; JONES \& TURNER, 1978). C'est ainsi qu'on peut observer (fig. $2 a$, b) qu'à la valeur du potentiel hydrique de $-1,4 \mathrm{MPa}$ correspond, chez Dichanthium, un déficit de saturation hydrique de 10 p. 100 en condition d'irrigation optimale et de 9 p. 100 sous contrainte hydrique et, chez Chrysopogon, des déficits de 17,5 p. 100 et 13,5 p. 100 . Le déficit de saturation hydrique correspondant à une valeur donnée du potentiel hydrique est donc nettement plus élevé chez Chrysopogon, ce qui conduit à penser que Dichanthium est plus adapté à la contrainte hydrique. Cette aptitude du Dichanthium à maintenir son état hydrique à un niveau plus favorable, sous contrainte, peut être attribuée à l'intervention d'un mécanisme physiologique. Le changement des relations hydrodynamiques qui caractérisent l'état hydrique foliaire, peut être dû à l'intervention de 2 mécanismes : l'ajustement osmotique et/ou la diminution d'élasticité de parois cellulaires (module d'élasticité élevé) (NoÏTSAKIS \& BERGER, 1984). Aucun élément ne permet de départager pour le moment ces 2 mécanismes chez Dichanthium. Une étude plus détaillée, au niveau de l'état hydrique cellulaire, pourrait apporter une solution à ce problème.

\section{B. Relations entre le potentiel hydrique $\Psi_{F}$ et les paramètres de production}

\section{Potentiel hydrique $\Psi_{F}$ et indice foliaire (I.F.)}

L'évolution de l'indice foliaire (I.F.) en fonction du potention hydrique $\Psi_{F}$ sous 2 régimes hydriques contrastés est sensiblement différente entre les 2 espèces (fig. 3a, b). L'I.F. chez Dichanthium commence à diminuer à partir de valeurs du potentiel hydrique sensiblement śgáles $(-1,2 ;-1,3 \mathrm{MPa})$ quel que soit le régime hydrique ; la diminution de l'I.F. chez Chrysopogon, au contraire, commence à partir de $-0,7 \mathrm{MPa}$ en conditions d'irrigation optimale et de $-1,0 \mathrm{MPa}$ sous contrainte hydrique à partir de valeurs de potentiel inférieures à - $1 \mathrm{MPa}$. Pour la même valeur du potentiel hydrique, l'indice foliaire chez Dichanthium est donc plus élevé que chez Chrysopogon, quel que soit le régime hydrique. La chute rapide de l'indice foliaire chez le Chrysopogon nous permet de supposer que celui-ci, par une réduction de sa surface foliaire, diminue progressivement sa consommation d'eau et atténue ainsi la contrainte hydrique effectivement subie, contrainte à laquelle il est moins résistant que Dichanthium (fig. 1a, b). Mais, à cette évolution de l'indice foliaire au-dessous de 4 ou 3, correspond une diminution de la production en biomasse (LARCHER, 1980 ; HANSON, 1971) plus forte chez Chrysopogon que chez Dichanthium.

\section{Potentiel hydrique foliaire $\Psi_{F}$ et taux d'élongation du limbe (T.E.L.)}

L'évolution du taux d'élongation du limbe en fonction du potentiel hydrique au cours d'une période de
20 jours courant juin dans 2 conditions d'alimentation hydrique est présentée dans les figures $4 \mathrm{a}, \mathrm{b}$. En conditions d'irrigation optimale, le taux d'élongation du limbe diminue rapidement lorsque le potentiel hydrique décroît et devient nul lorsque celui-ci atteint - 2,1 MPa chez Dichanthium et $-1,8 \mathrm{MPa}$ chez Chrysopogon. Sous contrainte hydrique, le taux d'élongation des feuilles décroît moins vite et s'annule pour des valeurs du potentiel hydrique égales à - 3,4 MPa chez Dichanthium et - 2,8 $\mathrm{MPa}$ chez Chrysopogon. CUTLER et al. (1980) ont également observé, chez Oryza sativa soumis à une contrainte hydrique rapide, que le taux d'élongation des limbes diminue en fonction du potentiel hydrique, moins vite chez les plantes stressées que chez les plantes bien irriguées. GRIMES \& YAMADA (1982) ont aussi établi une relation semblable entre le taux d'élongation des limbes et le potentiel hydrique. Dans le cas présent, pour une même valeur du potentiel hydrique, le taux d'élonga-

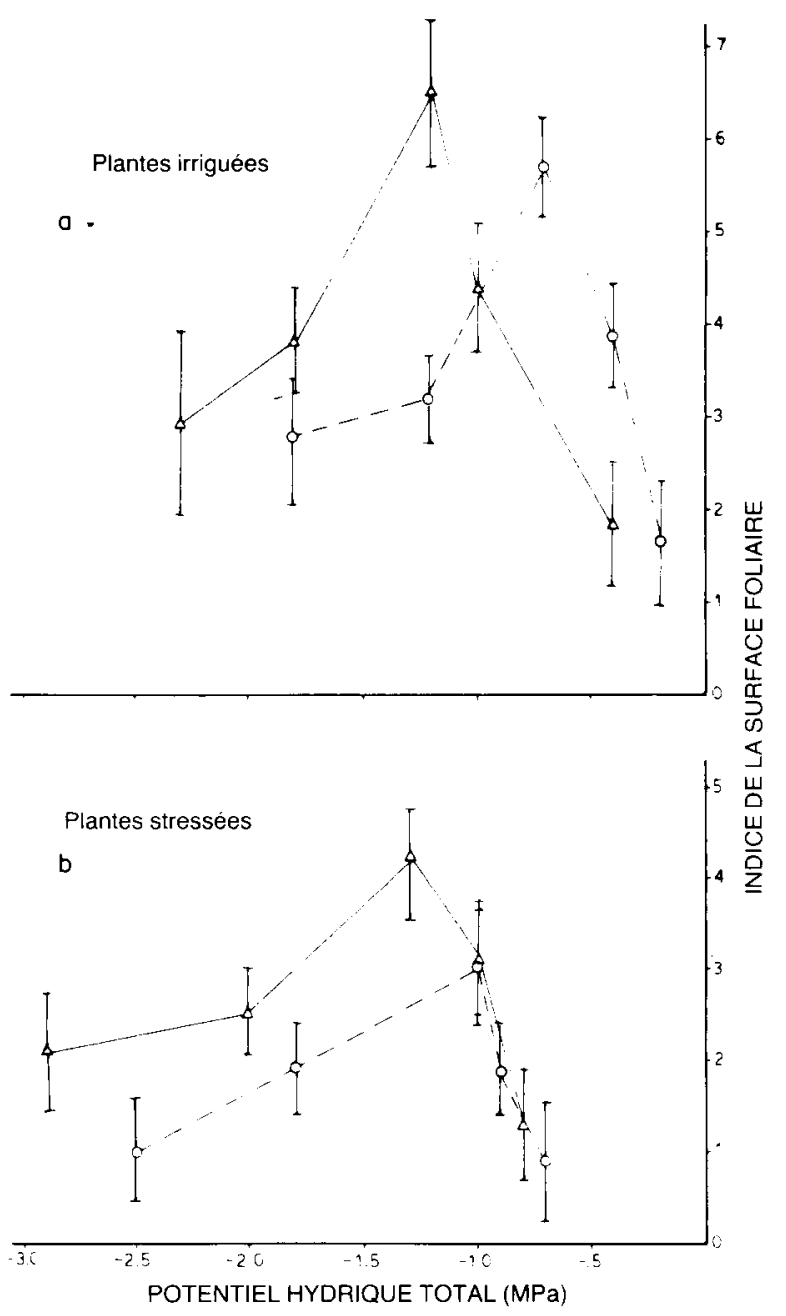

Figure 3

Evolution de l'indice de surface foliaire en fonction du potentiel hydrique foliaire chez Dichanthium ischaemum $(\Delta-\Delta)$ et chez Chrysopogon gryllus $(O-O)$.

a. En condition d'irrigation optimale.

b. Sous contrainte hydrique.

Les mesures étaient effectuées les 24/4, 8/5, 25/5, 19/6, 17/7.

L.A.I. change in relation to leaf water potential in Dichanthium ischaemum $(\Delta-\Delta)$ and Chrysopogon gryllus (O-O).

a. Under optimum irrigation conditions.

$b$. Under water stress.

Measurement dates : 24/4, 8/5, 25/5, 19/6, $17 / 7$. 


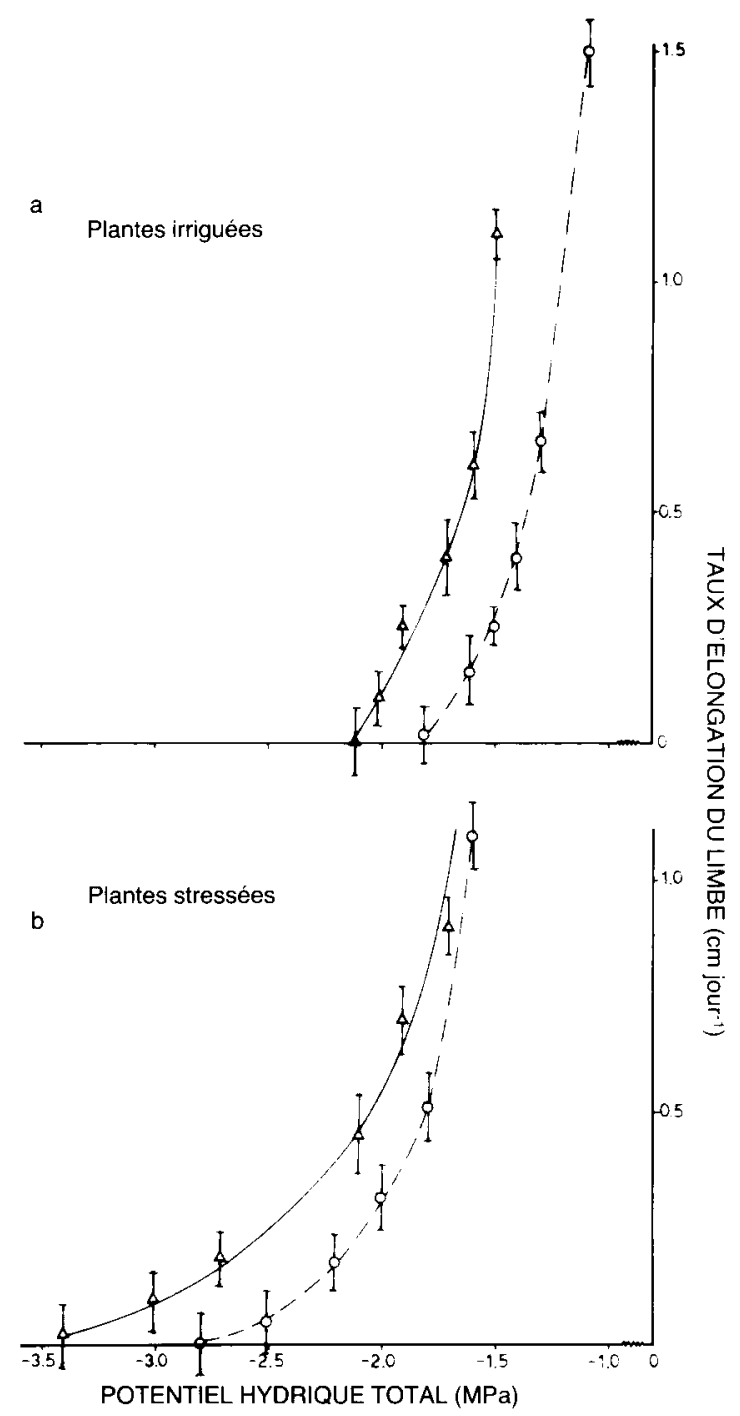

Figure 4

Evolution du taux d'élongation du limbe en fonction du potentiel hydrique foliaire mesuré au cours d'une période de 20 jours à partir du 5/6, chez Dichanthium ischaemum $(\Delta-\Delta)$ et chez Chrysopogon gryllus $(\mathrm{O}-\mathrm{O})$

a. En condition d'irrigation optimale.

b. Sous contrainte hydrique.

Les barres indiquent l'erreur standard du T.E.L.

Changes of leaf expansion rate in relation to leaf water potential for the period of 20 days from $5 / 6$ in Dichanthium ischaemum $(\Delta-\Delta)$ and Chrysopogon gryllus $(\mathrm{O}-\mathrm{O})$.

a. Under optimum irrigation conditions.

b. Under water stress.

tion des limbes est plus élevé chez les plantes stressées que chez les plantes irriguées et il est également plus élevé chez Dichanthium que chez Chrysopogon. Les plantes stressées sont donc moins sensibles que les autres à la diminution du potentiel hydrique et Dichanthium est la moins sensible des 2 espèces.

\section{Potentiel hydrique foliaire $\Psi_{F}$ et taux de croissance de la biomasse (T.C.B.)}

L'influence du déficit hydrique sur l'indice foliaire et le taux d'élongation des limbes est quantitativement intégrée dans la relation entre le potentiel hydrique et

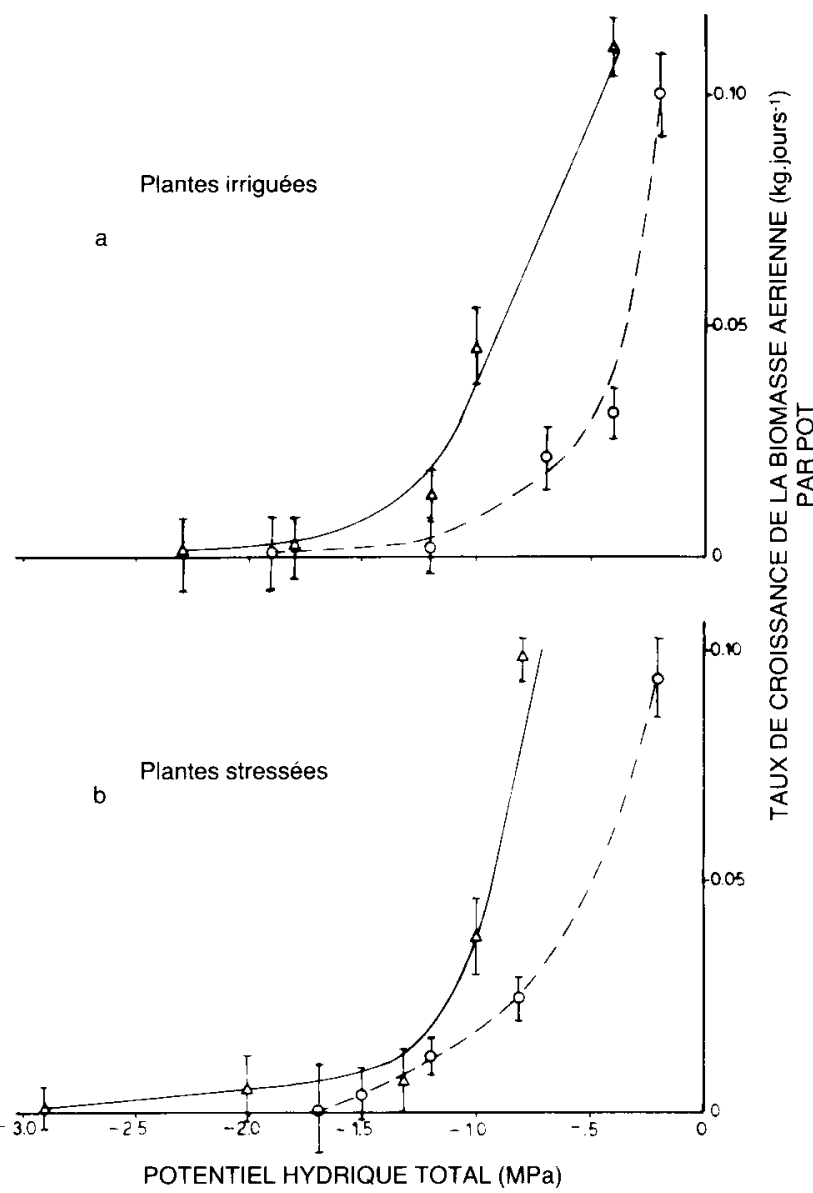

Figure 5

Evolution du taux de croissance de la biomasse aérienne en fonction du potentiel hydrique foliaire chez Dichanthium ischaemum et chez Chrysopogon gryllus.

a. En condition d'irrigation optimale.

b. Sous contrainte hydrique.

Les dates des mesures coincident avec celles de l'indice de surface foliaire.

Les barres indiquent l'erreur standard du T.C.B.

Changes of leaf relative growth rate in relation to leaf water potential in Dichanthium ischaemum $(\Delta-\Delta)$ and Chrysopogon gryllus $(\mathrm{O}-\mathrm{O})$.

a. Under optimum irrigation conditions.

$b$. Under water stress.

The measurement dates are the same as for L.A.I.

le taux de croissance de la biomasse (T.C.B.) (fig. 5a, b). L'allure du taux de croissance de la biomasse en fonction du potentiel hydrique est à peu près semblable à celle du taux d'élongation de limbes (fig. $4 a, b$ ). Le taux de croissance de la biomasse décroît sensiblement plus vite chez Chrysopogon que chez Dichanthium à partir de $-0,2$ et $-0,4 \mathrm{MPa}$ et il cesse pour $-1,2$ et $-2,4 \mathrm{MPa}$ chez Chrysopogon et Dichanthium respectivement. Sous contrainte hydrique, la diminution est plus lente et le taux de croissance s'annule à un niveau du potentiel hydrique encore plus faible, de l'ordre de - 1,7 chez Chrysopogon et $-2,9 \mathrm{MPa}$ chez Dichanthium. Une telle influence du potentiel hydrique sur le taux de croissance de la biomasse peut être considérée comme la combinaison de 2 effets, l'un concernant l'indice foliaire et l'autre le taux d'élongation des limbes, sur la production de biomasse. 


\section{CONCLUSIONS}

Les résultats obtenus dans le cadre de cette étude montrent que Dichantium manifeste une résistance à la sécheresse sensiblement plus élevée que celle de Chrysopogon, en ce qui concerne entre autres le taux de croissance de la biomasse aérienne. Ceci peut être lié aux propriétés hydrodynamiques des feuilles du Dichanthium qui semble maintenir un état hydrique foliaire plus favorable ce qui est incontestablement un élément important pour la valorisation des pâturages et pour leur exploitation rationnelle.
En outre, la grande sensibilité de l'elongation des feuilles chez les 2 espèces au déficit hydrique, au cours de leur cycle biologique, indique, que le taux d'élongation du limbe pourrait être un indice utile de la contrainte hydrique développée dans les tissus foliaires. Sur le plan pratique cet indice peut devenir un bon critère de la résistance à la sécheresse, utilisable dans un programme d'amélioration par voie génétique. Il faut souligner toutefois que ces résultats ont été obtenus sur les plantes en pots et risquent donc d'être modifiés en conditions de plein champ.

Reçu le 6 janvier 1987. Accepté le 8 octobre 1987.

\section{RÉFÉRENCES BIBLIOGRAPHIQUES}

Barrs H. D., 1968. Determination of water deficits in plant tissues. In : T. T. Kozlowski (ed.). Water Deficit and Plant Growth Vol. I Acad. Press, p. 236-368.

Begg J. E., Turner N. C., 1976. Crop water deficits. Adv. Agron. $28,161-217$

Berger A., 1978. L'alimentation en eau en milieu salé. Soc. Bot. Fr., Actualités Bot., 3/4, 159-176.

Boyer J. S., 1970. Leaf enlargement and metabolic rates in corn, soybean, and sunflower at various leaf water potentials. Plant Physiol., 46, 233-235.

Boyer J. S., McPherson H. G., 1975. Physiology of water deficits in cereal crops. Adv. Agron., 27, 1-23.

Cátsky J., 1965. Leaf disk method for determining water saturation deficit. UNESCO, Paris, Arid Zone Res., 25, 353-360.

Cutler J. M., Shahan K. W., Steponkos P. L., 1980. Influence of water deficits and osmotic adjustement of leaf elongation in rice. Crop Sci., 20, 314-318.

Grimes D. W., Yamada H., 1982. Relation of cotton growth and yield to minimum leaf water. Crop Sci., 22, 134-139.

Hanson W. D., 1971. Selection for differential productivity among juvenile maize plants : associated net photosynthetic rate and leaf area changes. Crop Sci., 11, 334-339.

Horst G. L., Nelson C. J., Asay K. H., 1978. Relationship of leaf elongation to forage yield of tall fescue genotypes. Crop Sci., 18, 715-719.

Hoveland C. S., Foutch H. W., Buchanan G. A., 1974. Responses of Phalaris genotypes and other cool-season grasses to temperature. Agron. J., 66, 686-690.

Hsiao T. C., 1973. Plant responses to water stress. Ann. Rev. Plant Physial., 24, 519-570.

Hsiao T. C., Ferere B., Acevedo E., Handerson D. W., 1976. Water stress and dynamics of growth and yield of crop plant. In : O. L. Lance, L. Kappen. E. D. Schulze (eds). Water and plant Life. Problems and Modern Approaches Spring-Verlag, Berlin, p. 281305.

Jones W. X., Turner L. C., 1978. Osmotic adjustment in leaves of Sorghum in response to water deficit. Plant Physiol., 61, 122-126.
King M. J., Bush L. P., 1985. Growth and water use of tall fescue as influenced by several soil drying cycles. Agron. J., 77, 1-4.

Larcher W., 1980. Physiological Plant Ecology. Springer-Verlag, Berlin Heidelberg, New York, p. 303

Ludlow M. M., 1976. Ecophysiology of $C_{4}$ grasses. In : O. L. Lance, L. Kappen, E. D. Schulze (eds). Water and Plant Life: Problems and Modern Approaches. Springer-Verlag, Berlin, p. 364 368.

Ludlow M. M., Ng T. T.. 1977. Leaf elongation rate in Panicum maximum var. trichoglume following removal of water stress. Aust. J. Plant Plysiol., 4, 263-272.

Noïtsakis B., Berger A.. 1984. Relations hydriques chez Dactylis glomerata et Dichanthium ischaemum cultivés sous deux régimes hydriques contrastés $O E$ col. Plant., 5, 75-88.

Radford P. J., 1967. Growth analysis formulae. Their use and abuse. Crop Sci., 7. 171-175.

Ritchie G. A., Hinckley T. M., 1975. The pressure chambre as an instrument for ecological research. In : A. Macfadyen (ed.). Advances in Ecological Research. Acad. Press, V9 ; p. 165-254.

Sanchez-Diaz M. F., Kramer P. J., 1971. Behavior of corn and sorghum under water stress and during recovery. Plant Physiol., 48 613-616.

Scholander P. F., Hammel H. T., Brastreet E. D., Hemmingsen E. A., 1965. Sap pressure in vascular plant. Science, 148, 339-346.

Thomas J. C., Brown K. W., Jordan W. R., 1976. Stomatal responses to leaf water potential as affected by preconditioning water stress in the field. Agron. J., 68, 706-708.

Turner N. C., 1979. Drought resistance and adaptation to water deficits in crop plants. In: H. Mussel, R. Staples (eds). Stress Physiology in Crop Plants. John Wiley and Sons., p. 343-372.

Turner N. C., 1981. Techniques and experimental approaches for the measurement of plant water status. Plant and Soil, 58, 339-366.

Wolf D. D., Parrish, D. J., 1982. Short-term growth responses of tall fescue to changes in soil water potential and to defoliation. Crop Sci., 22, 996-999. 